\title{
Intensity-modulated radiation therapy for early-stage breast cancer: is it ready for prime time?
}

This article was published in the following Dove Press journal:

Breast Cancer - Targets and Therapy

20 March 2017

Number of times this article has been viewed

\section{Tabitha Y Chan \\ Poh Wee Tan \\ Johann I Tang}

Department of Radiation Oncology, National University Cancer Institute, Singapore, Singapore
Correspondence: Tabitha Y Chan Department of Radiation Oncology, National University Cancer Institute, Singapore, 5 Lower Kent Ridge Road, SI 19074, Singapore Email chantabitha@gmail.com

\begin{abstract}
Whole breast external beam radiotherapy (WBEBRT) is commonly used as an essential arm in the treatment management of women with early-stage breast cancer. Dosimetry planning for conventional WBEBRT typically involves a pair of tangential fields. Advancement in radiation technology and techniques has the potential to improve treatment outcomes with clinically meaningful long-term benefits. However, this advancement must be balanced with safety and improved efficacy. Intensity-modulated radiation therapy (IMRT) is an advanced technique that shows promise in improving the planning process and radiation delivery. Early data on utilizing IMRT for WBEBRT demonstrate more homogenous dose distribution with reduction in organs at risk doses. This translates to toxicities reduction. The two common descriptors for IMRT are forward-planning "fields in field" and inverse planning. Unlike IMRT for other organs, the aim of IMRT for breast planning is to achieve dose homogeneity and not organ conformality. The aim of this paper was to evaluate whether IMRT is ready for prime time based on these three points: 1) workload impact, 2) the clinical impact on the patient's quality of life, and 3) the appropriateness and applicability to clinical practice.
\end{abstract}

Keywords: early-stage breast cancer, radiotherapy, intensity-modulated radiation therapy, workload impact, quality of life, clinical practice

\section{Introduction}

Breast cancer is the most common cancer that occurs in females and the majority of cases are comprised of early-stage disease. ${ }^{1}$ Breast conserving surgery (BCS), followed by adjuvant radiation therapy (RT), is an essential arm in the treatment management of women with early-stage breast cancer. ${ }^{2}$ Onitilo et $\mathrm{al}^{3}$ demonstrated that the judicious use of adjuvant RT post-BCS improves survival outcomes compared to radical mastectomy alone. ${ }^{3}$ Adjuvant RT also halves the recurrence rate thereby significantly improving survival rates. ${ }^{4,5}$ This translates to more women at risk of developing chronic toxicities associated with their treatment management.

Whole breast external beam radiotherapy (WBEBRT) is associated with acute RT-induced toxicities such as skin desquamation, edema, and pain. This can affect patient's quality of life (QoL) and also influence their decision regarding the use of BCS with adjuvant RT. ${ }^{6}$ Hence, the ability to ensure treatment efficacy while reducing toxicities is increasingly important.

Advancement in radiation technology and techniques has the potential to improve treatment outcomes with clinically meaningful long-term benefits. ${ }^{7}$ However, this advancement must be balanced with safety and improved efficacy. As such, breast RT has progressed from two-dimensional (2D) treatment therapy, based on anatomical 
landmarks and conventional simulator to three-dimensional (3D) therapy that utilizes computed tomography planning.

Dosimetry planning for conventional WBEBRT typically involves a pair of tangential fields directed to the breast at an angle. The tumor volume or target is the whole ipsilateral breast and the usual field borders are superiorly from the clavicular head to the infra-mammary fold inferiorly and medially from the mid-sternum to the mid-axillary line laterally. A drawback of this technique is the propensity for a more inhomogeneous distribution of the radiation dose due to varying breast shapes and sizes. Due to the conical shape of the breast, the radiation beam must travel through more tissue along the chest wall compared to the nipple areolar complex (NAC) region. This results in significantly higher doses (hot spots) in the NAC region. To improve dose homogeneity throughout the entire breast, wedges as well as additional "fields-in-field" methods may be utilized. ${ }^{8}$ WBEBRT is often delivered without explicit contouring of the target volume. ${ }^{9}$

\section{What is intensity-modulated radiation therapy (IMRT)?}

Technical advances in radiation oncology rest on the principle of optimizing the therapeutic ratio, that is delivering maximum radiation dose to the target volume while minimizing the dose to the surrounding normal tissues (also known as organs at risk [OAR]), thereby minimizing tissue toxicity. IMRT is an advanced radiation planning and delivery technique that has shown to be able to achieve a greater therapeutic ratio. ${ }^{10,11}$ However, IMRT has a few disadvantages: planning time is operator dependent and based on the planner's experience, treatment time is longer, and in most countries, IMRT represents a more expensive treatment. These disadvantages, despite its proven clinical advantage of a greater therapeutic ratio, have resulted in a slow uptake for IMRT, making conventional radiation therapy (CRT) the more popular choice in most centers. ${ }^{12}$

IMRT is possible due to advances in multileaf collimator (MLC) technology. Within the linear accelerator collimator head lie multiple leaves whose purpose is to shape the beam. By varying the shape of the beam or the speeds at which the MLC leaves travel, the radiation beam is effectively shaped to vary the dose distribution to the target volume.

\section{Types of breast IMRT techniques}

The two common descriptors for IMRT are forward-planning "fields in field" (FP FIF) and inverse planning (IP). The aim of IMRT for breast planning is to achieve dose homogeneity and not organ conformality. ${ }^{13}$
FP FIF IMRT is a technique where limited numbers of MLC segments are determined. ${ }^{14}$ Beams eye view, when viewed from the radiation beam's perspective achieved by complex computer algorithm, is utilized to provide a $3 \mathrm{D}$ visualization of the isodose distribution from open unmodulated fields (Figure 1). A limited number of MLC segments with optimal shape and weightings are then determined. These static MLC segments are then delivered sequentially by a step and shoot technique.

IP is an IMRT technique that requires contouring of target volumes and OARs. ${ }^{15}$ This way, a homogenous dose distribution can be achieved and controlled by applying constraints to the target volume and OAR. IP IMRT is often delivered using sliding window dynamic multileaf collimation that allows superior spatial intensity resolution throughout the target volume. ${ }^{15}$

Both FP FIF and IP IMRT techniques achieve comparable excellent dose distribution. ${ }^{16}$ The aim of this paper was to evaluate whether IMRT is ready for prime time based on the following three points: 1) workload impact, 2) clinical impact on the patient's QoL, and 3) appropriateness and applicability to clinical practice.

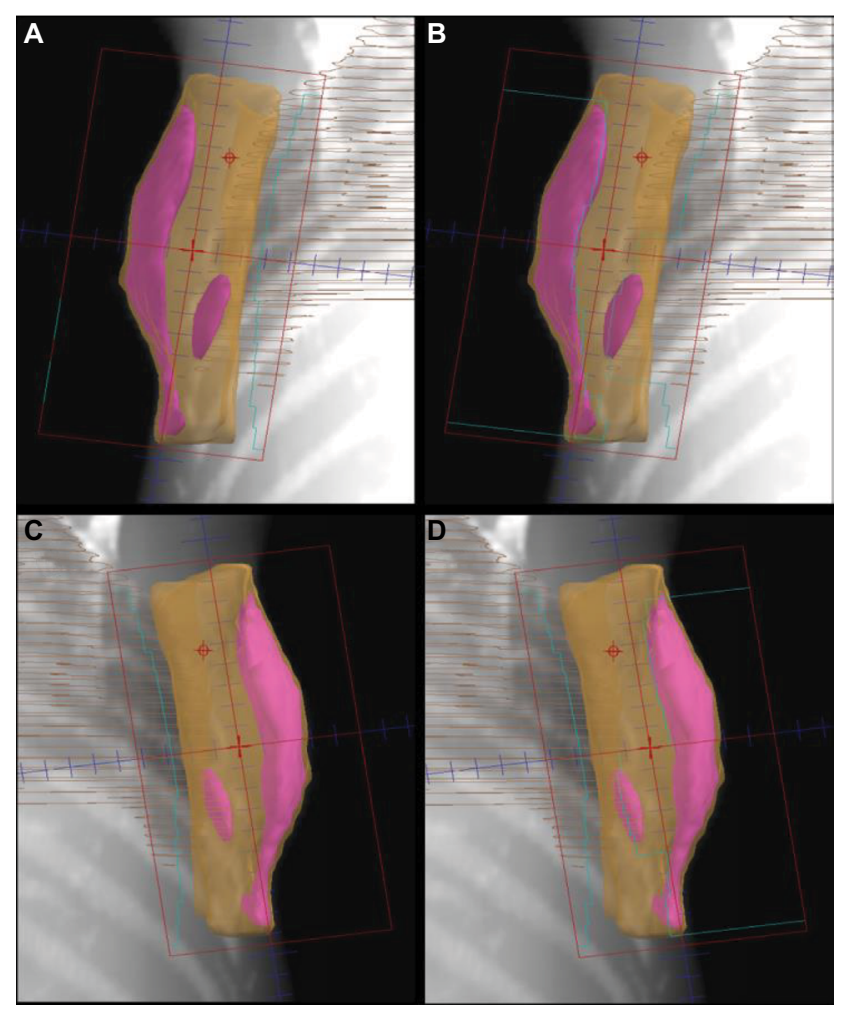

Figure I Forward-planning "field in field" IMRT technique. A and C demonstrate tangent fields without shielding. $\mathbf{B}$ and $\mathbf{D}$ demonstrate tangent fields that shielded the volumes receiving $\geq 110 \%$ of the prescribed dose using multileaf collimator. Abbreviation: IMRT, intensity-modulated radiation therapy. 


\section{Workload impact}

The efficiency of resources must be ascertained as it impacts the workload and the human resource required. These parameters can be used to compare the impact of CRT and IMRT treatment techniques on resources: 1) time to plan, 2) time to deliver treatment, and 3) time to perform quality assurance (QA).

All planning timings stated in the following excluded contouring timings.

The Cambridge Breast IMRT Trial is a randomized control trial (RCT) comparing 3D or FP FIF IMRT to 2D planning of two-field tangents for 815 randomized patients. ${ }^{17}$ The median planning time was four times longer for 3D or FP FIF IMRT compared to 2D planning (3D or FP FIF IMRT vs 2D planning: 90 minutes vs 20 minutes). The median treatment time for 3D or FP FIF IMRT compared to 2D tangents was 12.7 minutes and 9.4 minutes, respectively.

Al-Rahbi et a $1^{15}$ compared the planning times of 3D CRT, FP FIF IMRT, and IP IMRT. ${ }^{15}$ The planning times were 20-30 minutes, 15-20 minutes, and 50-60 minutes, respectively. However, planning times are subjective as it is dependent on dosimetrist's experience. Treatment delivery timings (inclusive of patient setup) for 3D CRT, FP FIF IMRT, and IP IMRT were 10-15 minutes, 10-15 minutes, and 15-20 minutes, respectively.

A possible explanation of why the planning timings of 3D CRT and IMRT in Al-Rahbi et al ${ }^{15}$ study is substantially less than the Cambridge Breast IMRT Trial is that breast IMRT planning in the Cambridge Breast IMRT Trial was at its infancy. ${ }^{17}$ As technology and planning experience matures, planning timings may consequentially be shortened.

IMRT planning has evolved from FP FIF IMRT to hybrid IMRT. Several studies have demonstrated that hybrid IMRT achieved comparable dosimetric outcomes as FP FIF IMRT with comparable treatment planning time. ${ }^{18-21}$

Descovich et $\mathrm{al}^{18}$ compared a hybrid direct aperture optimized (hDAO) IP IMRT to FP FIF IMRT for WBEBRT. ${ }^{18}$ Both hDAO IP IMRT and FP FIF IMRT utilized two tangential beam directions. hDAO IP IMRT plans were inversely optimized and utilized two open fields with eight segments in two tangential beam directions. Both plans achieved comparable breast coverage and OAR sparing. hDAO IP IMRT plans outperformed FP FIF IMRT plans in terms of reduction of breast volume receiving $105 \%$ of prescribed dose in the hDAO IP IMRT than in the FP FIF IMRT plans: $25 \%$ vs $63 \%$ ( $p=0.008$ ) for small, $22 \%$ vs $57 \%$ $(p=0.005)$ for medium, and $28 \%$ vs $53 \%(p=0.005)$ for large breasts. Lumpectomy coverage was marginally better for hDAO IP IMRT compared to FP FIF IMRT ( $92.4 \%$ vs
90.9\%). The planning time for hDAO IP IMRT plans was substantially shorter compared to FP FIF IMRT (60-90 minutes vs $20-30$ minutes).

Farace et al ${ }^{19}$ similarly compared tangential and nontangential beam arrangements for hybrid IP IMRT plans utilizing a semi-automated method and a plan optimization volume set as a target objective during IP. ${ }^{19}$ The tangential beam arrangement was a four-field technique (two conventional open plus two IP IMRT tangents), and the non-tangential beam arrangement was a six-field technique where two non-tangential (anterior-oblique) IP IMRT beams were added. All dosimetric goals were met; the dosimetry was similar between the tangential and non-tangential beam arrangements and the average planning time was $10 \mathrm{~min}$ utes. Both studies demonstrated that the merits of utilizing hybrid IP IMRT lie in its largely automated process while achieving a comparable plan quality to FP FIF IMRT yet reducing planning time, reducing manual process during planning as well as dependence on dosimetrist's expertise. The limited monitor units (MU) contribution from IP IMRT beams allowed QA to be less taxing compared to full IP IMRT. ${ }^{19}$ An added advantage of utilizing hybrid IP IMRT is the use of open fields as a main radiation source as this makes planned dosimetry less sensitive to changes in breast shape and setup inaccuracies. ${ }^{22}$

Overall, utilizing IMRT, regardless of the technique used, lengthens the treatment planning time and QA time required. However, the outcome is a plan with better dosimetry and a more homogenous distribution.

\section{Clinical impact on patient's QoL}

Side effects due to RT affect multiple dimensions of QoL. They cause pain, emotional distress, and affect day-to-day functioning. ${ }^{23}$ RCTs of FP FIF IMRT vs conventional tangents for breast cancer demonstrated that plans with improved dosimetry correlate with reduced skin toxicities that directly affect cosmesis, pain, and QoL. ${ }^{10,24-26}$

The Royal Marsden trial is a prospective RCT that compared the 2D standard tangents of FP FIF IMRT. ${ }^{25}$ Three hundred and six women were randomized into FP FIF IMRT (test arm) or 2D standard tangents (control arm) after BCS. All were prescribed WBEBRT with 50 Gy followed by 10 Gy electron boost. The endpoints included breast cosmesis, pain, and QoL. This trial found that greater normal tissue changes were correlated to increasing breast size and distortion of breast shape post-surgery, which resulted in greater dose inhomogeneity. However, dose homogeneity was improved with FP FIF IMRT. 2D standard tangents had greater dose 
inhomogeneity with a larger proportion of dose exceeding $110 \%$ compared to FP FIF IMRT technique. This was found to correlate with a higher incidence of late adverse events for 2D standard tangents compared to FP FIF IMRT technique.

When comparing the proportion of patients showing changes in breast appearance at 1, 2, and 5 years after randomization by maximum dose absorbed by breast, there was a significant difference in the type of breast changes with the absence or presence of doses exceeding 105\%. Sections of breast exceeding $105 \%$ dose were 1.9 times ( $95 \%$ confidence interval $[\mathrm{CI}] 1.3-2.9, p=0.002$ ) more likely to have breast changes than sections not exceeding $105 \%$ dose. After adjusting for the 2D standard tangents, the absence or presence of doses $>105 \%$ and its correlation to breast change remained significant ( $p=0.030)$ with odds ratio (OR) of $2.6(95 \% \mathrm{CI}$ 1.1-6.0). However, the significant treatment effect, that is dependent on the technique used, disappeared. This validates the rationale for correlating the statistical difference in incidence of the endpoints of the two techniques to dosimetry. It also validates the relationship between dosimetry and treatment effects and demonstrates that the more the changes, the longer the follow-up. The results favor FP FIF IMRT.

The Canadian Phase III multicenter trial is another prospective RCT with a similar design and prescription. However, this trial included and stratified all breast sizes - small, medium, and large. ${ }^{26}$

The trial arm was FP FIF IMRT, and the control arm was standard conventional tangents. Endpoints were acute skin reaction, pain, and QoL. The Canadian trial demonstrated that FP FIF IMRT significantly improved dosimetry. This correlated to a lower proportion of patients experiencing moist desquamation (FP FIF IMRT vs standard treatment: $31.2 \%$ vs $47.8 \%, p=0.002)$. In a multivariate analysis, smaller breast sizes $(p<0.001)$ and the use of FP FIF IMRT $(p=0.030)$ were significantly associated with a decreased risk of moist desquamation. Although the use of FP FIF IMRT did not correlate with pain and QoL, the presence of moist desquamation significantly correlated with pain $(p=0.002)$ and a reduced QoL $(p=0.003)$. Updated results on QoL showed that the benefits of IMRT was greatest in older women. ${ }^{27}$

The Cambridge IMRT trial is a RCT of FP FIF IMRT vs 2D standard tangents for early breast cancer. ${ }^{17}$ A total of 815 women with inhomogeneity exceeding 107\% were randomized. This trial demonstrated that dosimetry for all breast sizes benefits from FP FIF IMRT. In the 5-year follow-up following the Cambridge trial, toxicities such as overall cosmesis, breast shrinkage, and telangiectasia were compared between both arms..$^{28}$ Univariate analysis reported that FP FIF
IMRT arm had fewer suboptimal cosmesis (OR, 0.68; 95\% CI $0.48-0.96 ; p=0.027$ ) and skin telangiectasia (OR, 0.58; $95 \%$ CI $0.36-0.92 ; p=0.021)$. Although breast shrinkage was similar in both arms, the benefit of FP FIF IMRT for overall cosmesis $(p=0.038)$ and skin telangiectasia $(p=0.031)$ was significant. These results demonstrate that improved dose homogeneity with simple FP FIF IMRT correlates with a risk reduction of skin telangiectasia and superior overall cosmesis.

To date, there are no RCTs comparing IP IMRT with 3D CRT. However, two separate comparative dosimetric studies - which generated two plans, 3D CRT and IP IMRT, for each computed tomography data set - demonstrated that IP IMRT resulted in superior dose conformity with a larger volume receiving low dose. ${ }^{29,30}$ Hence, it could be expected that IP IMRT can reduce toxicities and improve overall cosmesis.

\section{Appropriateness and applicability to clinical practice}

To ascertain the appropriateness and applicability of breast IMRT as standard clinical practice, the following points should be duly considered (Table 1).

The planning objectives for CRT and IMRT are similar as $95 \%$ of the prescribed dose should cover $100 \%$ of the target volume and OAR constraints for both techniques must be respected. However, IMRT is more complex as deliberate attempts must be made to reduce dose homogeneity and lower the maximum dose. ${ }^{15,16}$

In terms of MUs, Al-Rahbi et $\mathrm{al}^{15}$ demonstrated that IP IMRT technique required more MUs compared to 3D CRT and FP FIF IMRT techniques. ${ }^{15}$ The number of MUs for the FP FIF IMRT technique was four times lesser than that for the IP IMRT technique (293 MU vs 1160 MU), whereas those for the 3D CRT technique was three times lesser (443 MU vs $1160 \mathrm{MU})$. The number of MU for 3D CRT was higher than that for the FP FIF IMRT technique (443 MU vs 293 MU) because of the use of wedges in the 3D CRT technique. A separate study comparing forward and inverse planning methods demonstrated that IP IMRT required the most number of MUs, as per the following results: CRT using physical wedges: $278 \pm 15$; CRT using dynamic wedges: $235 \pm 10$; FP FIF IMRT: $227 \pm 9$; and IP IMRT: $437 \pm 84 .{ }^{31}$ This inevitably increased treatment time. ${ }^{15,31}$ Other implications such as higher machine leakage and whole body dose may increase the risk of radiation-induced secondary malignancy. ${ }^{15,31}$

The literature reports that IMRT requires longer planning time and advanced planning skills. ${ }^{32}$ Smith et $\mathrm{al}^{32}$ found that CRT required a mean time of 15 minutes 
Table I Summary of the points of comparison between CRT and IMRT

\begin{tabular}{|c|c|c|c|}
\hline \multirow[t]{2}{*}{ References } & \multirow[t]{2}{*}{ Points of consideration } & \multicolumn{2}{|l|}{ Techniques } \\
\hline & & CRT & IMRT (FP FIF or IP) \\
\hline Al-Rahbi et $\mathrm{a}^{15}$ and Nguyen et al ${ }^{16}$ & Planning objectives & Simple & Complex \\
\hline Al-Rahbi et al $\left.\right|^{15,31}$ & MU & More MU $\rightarrow$ more scatter & Fewer MU $\rightarrow$ less scatter \\
\hline Al-Rahbi et al ${ }^{15,31}$ & Beam on time & Less & More \\
\hline Al-Rahbi et al ${ }^{15}$ and Smith et $\mathrm{a}^{32}$ & Dosimetrist skill & Less & More \\
\hline Al-Rahbi et $\mathrm{al}^{15}$ and Smith et $\mathrm{a}^{32}$ & Planning time & Less & More \\
\hline Al-Rahbi et $\mathrm{al}^{15}$ & QA time & Less & More \\
\hline George et $\mathrm{al}^{33}$ and Jain et $\mathrm{al}^{34}$ & $\begin{array}{l}\text { Susceptibility to breast deformation } \\
\text { and intrafraction movement }\end{array}$ & Less susceptible & More susceptible \\
\hline Alford et $\mathrm{a}^{35}$ and Askoxylakis et a ${ }^{36}$ & Boost: sequential vs simultaneous & $\begin{array}{l}\text { Sequential } \rightarrow \text { less conformality } \\
\text { to boost volume }\end{array}$ & $\begin{array}{l}\text { Simultaneous } \rightarrow \text { greater } \\
\text { conformality to boost volume }\end{array}$ \\
\hline
\end{tabular}

Abbreviations: CRT, conventional radiation therapy; MU, monitor units; QA, quality assurance; IMRT, intensity-modulated radiation therapy; FP FIF, forward-planning "fields in field"; IP, inverse planning.

compared to 60-90 minutes required by FP FIF IMRT, while Al-Rahbi et $\mathrm{al}^{15}$ reported an average time of 20-30 minutes, 15-20 minutes, and 45-60 minutes for 3D CRT, FP FIF IMRT, and IP IMRT, respectively. It is interesting to note that the planning time for FP FIF IMRT is quartered in Al-Rahbi et al's ${ }^{15}$ study. This might be attributed to the fact that as technology matures, the time taken for planning is shortened as the planning system becomes more powerful. Also, compared to 3D CRT and FP FIF IMRT, IP IMRT requires patient-specific QA measurements. ${ }^{15}$ This additional QA time must be taken into account when measuring the total workload per plan. Compared to the IP IMRT and 3D CRT plans, the higher-quality FP FIF IMRT plans are likely to be generated in a shorter time without requiring a high level of planning ability. ${ }^{15}$

An earlier concern with breast IMRT is its susceptibility to intrafraction movement during treatment and breast shape changes due to the interplay between MLC and respiratory motion during treatment and the breast shape, respectively. ${ }^{33}$ However, it is interesting to note that both an early study by George et $\mathrm{al}^{33}$ and a separate study by Jain et $\mathrm{al}^{34}$ demonstrated no statistically significant differences between the planned and expected dose distributions in any phase of the respiratory cycle during treatment suggesting that breast deformity and intrafraction movement have limited impact. ${ }^{33,34}$

Another advantage of IMRT is that a simultaneous integrated boost dose (SIB) can be delivered simultaneously compared to the widely used sequential electron boost. Improvements in skin sparing, cosmesis, and dose conformality are expected. This is especially useful for deep-seated boost volumes. Total dose and dose per fraction to OAR are also decreased. This also shortens the overall treatment time for patients and reduces planning workload. However, SIB can only be achieved with IP IMRT. ${ }^{35,36}$

\section{Discussion}

IMRT is gaining widespread acceptance across many centers. Resource limitation is a concern that may hinder the adoption of IMRT. Hence, selecting the planning technique is often a trade-off among four factors: 1) plan quality; 2) time, expertise, and effort to generate a plan; 3) need for QA; and 4) beam on time. As early-stage breast cancer makes up the bulk of patient proportion in a typical radiotherapy center, any increase in treatment complexity will affect resource allocation. A systematic review by $\mathrm{Chen}$ et $\mathrm{al}^{37}$ found a relative risk of 1.11 (95\%CI 1.04-1.19) of local recurrence per month of waiting time for postoperative RT for breast cancer. ${ }^{37}$ Thus, it could be inferred that treatment delay as a result of more complex planning techniques could pose a risk for patients.

This is especially relevant to IP IMRT as it requires physics QA of the MU calculation, deliverability, and linear accelerator (linacs), which adds a strain to physics resources and may limit linacs that can be used for these treatments too.

Although the literature suggests that IMRT allows the rational use of current resources with a slight increase in planning expertise, it remains unlikely that every patient requires IMRT, both IP and FP FIF. This necessitates the judicious selection of breast cancer patients who would most benefit from IMRT. This may help mitigate the impact on clinic resources.

\section{Conclusion}

The merit of breast IMRT is well documented. However, the routine application of breast IMRT has to be wisely considered. Conventional tangent for WBEBRT remains simple and effective. Advanced technology such as IMRT, albeit with homogeneity and toxicity benefits, may further strain the health care system already fraught with limited clinical resources and time constraints. This is especially relevant for 
breast RT that represents a substantial portion of a typical radiation oncology center.

The definition of IMRT for breast remains ambiguous. It is imperative that a clear distinction should be made between FP FIF IMRT and IP IMRT. The distinct difference is that FP FIF IMRT, although popularly termed IMRT, should be more accurately defined "field in field" 3D CRT (FIF 3D CRT). These two different descriptors essentially refer to the same technique that shares the same goal of achieving dose homogeneity within the breast to reduce skin toxicities, thereby preserving or improving cosmesis. The secondary goal is for better OAR sparing especially for the heart in left breast cancer patients.

Both patients and physicians alike can be misled that IP IMRT is superior to FP FIF IMRT, which is essentially FIF 3D CRT. The medical literature does not support this. While IP IMRT provides superior outcomes for other sites, IP IMRT for breast RT is not routinely advantageous compared to FIF 3D CRT. However, IP IMRT can be a useful tool for women with atypical anatomy such as severe pectus excavatum. ${ }^{38}$ Although IMRT for early-stage breast cancer is ready for prime time, incorporating IP IMRT as standard practice is not recommended as it does not always improve dose homogeneity or patient outcomes. Rather, the judicious selection of each technique's dosimetric advantage and treatment efficacy should be matched with the patient's characteristics.

\section{Disclosure}

The authors report no funding sources and no conflicts of interest in this work.

\section{References}

1. WHO. Fact Sheets by Cancer. Available from: http://globocan.iarc.fr/ Pages/fact_sheets_cancer.aspx. Accessed April 12, 2016.

2. American Cancer Society. Radiation for Breast Cancer. 2016. Available from: http://www.cancer.org/cancer/breastcancer/detailedguide/breastcancer-treating-radiation. Accessed November 7, 2016.

3. Onitilo AA, Engel JM, Stankowski RV, Doi SA. Survival comparisons for breast conserving surgery and mastectomy revisited: community experience and the role of radiation therapy. Clin Med Res. 2015;13(2):65-73.

4. Darby S, McGale P, Correa C, et al; Early Breast Cancer Trialists' Collaborative Group (EBCTCG). Effect of radiotherapy after breastconserving surgery on 10-year recurrence and 15-year breast cancer death: meta-analysis of individual patient data for 10,801 women in 17 randomised trials. Lancet. 2011;378(9804):1707-1716.

5. Kanapuru B, Ershler WB, Hesdorffer C, Jemal A, Yates JW. Long-term survival of older breast cancer patients: population-based estimates over three decades. Breast Cancer Res Treat. 2012;134(2):853-857.

6. Fisher J, Scott C, Stevens R, et al. Randomized phase III study comparing best supportive care to biafine as a prophylactic agent for radiationinduced skin toxicity for women undergoing breast irradiation: radiation therapy oncology group (RTOG) 97-13. Int J Radiat Oncol Biol Phys. 2000;48(5):1307-1310.

7. Kron T, Chua B. Radiotherapy for breast cancer: how can it benefit from advancing technology? Euro Med J. 2014;2:83-90.
8. Stefanovski Z, Smichkoska S, Petrova D, Lazarova E. Advantages of the technique with segmented fields for tangential breast irradiation. 2016; 75-78. Available from: https://inis.iaea.org/search/search. aspx?orig_q=RN:45026292. Accessed April 13, 2016.

9. Afkhami-Ardakani M, Esfahani M, Nourollahi Y, Ashuri-Taziyani Y, Hagh-Parast M. Assessment of the effect of breast size on dose distribution for 3D and conventional methods with TLD dosimetry in breast phantoms. 2015. Available from: http://fbt.tums.ac.ir/index.php/fbt/ article/view/39/36. Accessed November 8, 2016.

10. Harsolia A, Kestin L, Grills I, et al. Intensity-modulated radiotherapy results in significant decrease in clinical toxicities compared with conventional wedge-based breast radiotherapy. Int J Radiat Oncol Biol Phys. 2007;68(5):1375-1380.

11. Vicini FA, Sharpe M, Kestin L, et al. Optimizing breast cancer treatment efficacy with intensity-modulated radiotherapy. Int J Radiat Oncol Biol Phys. 2002;54(5):1336-1344.

12. Wang EH, Mougalian SS, Soulos PR, et al. Adoption of intensity modulated radiation therapy for early-stage breast cancer from 2004 through 2011. Int J Radiat Oncol Biol Phys. 2015;91(2):303-311.

13. McCormick B, Hunt M. Intensity-modulated radiation therapy for breast: is it for everyone? Semin Radiat Oncol. 2011;21(1):51-54.

14. Ozyigit G. Current role of modern radiotherapy techniques in the management of breast cancer. World J Clin Oncol. 2014;5(3):425-439.

15. Al-Rahbi ZS, Al Mandhari Z, Ravichandran R, et al. Dosimetric comparison of intensity modulated radiotherapy isocentric field plans and field in field (FIF) forward plans in the treatment of breast cancer. J Med Phys. 2013;38(1):22-29.

16. Nguyen S, Cao F, Ramaseshan R, et al. Template-based breast IMRT planning for increased workload efficiency. Radiat Oncol. 2013;8:67.

17. Barnett GC, Wilkinson J, Moody AM, et al. A randomised controlled trial of forward-planned radiotherapy (IMRT) for early breast cancer: baseline characteristics and dosimetry results. Radiother Oncol. 2009;92(1):34 41.

18. Descovich M, Fowble B, Bevan A, Schechter N, Park C, Xia P. Comparison between hybrid direct aperture optimized intensity-modulated radiotherapy and forward planning intensity-modulated radiotherapy for whole breast irradiation. Int J Radiat Oncol Biol Phys. 2010;76(1):91-99.

19. Farace P, Zucca S, Solla I, et al. Planning hybrid intensity modulated radiation therapy for whole-breast irradiation. Int J Radiat Oncol Biol Phys. 2012;84(1):e115-e122.

20. Fong A, Bromley R, Beat M, Vien D, Dineley J, Morgan G. Dosimetric comparison of intensity modulated radiotherapy techniques and standard wedged tangents for whole breast radiotherapy. J Med Imaging Radiat Oncol. 2009;53(1):92-99.

21. Mayo CS, Urie MM, Fitzgerald TJ. Hybrid IMRT plans-concurrently treating conventional and IMRT beams for improved breast irradiation and reduced planning time. Int J Radiat Oncol Biol Phys. 2005;61(3):922-932.

22. van Mourik A, van Kranen S, den Hollander S, Sonke JJ, van Herk M, van Vliet-Vroegindeweij C. Effects of setup errors and shape changes on breast radiotherapy. Int J Radiat Oncol Biol Phys. 2011;79(5):1557-1564.

23. Schnur JB, Ouellette SC, Dilorenzo TA, Green S, Montgomery GH. A qualitative analysis of acute skin toxicity among breast cancer radiotherapy patients. Psychooncology. 2011;20(3):260-268.

24. Barnett GC, Wilkinson JS, Moody AM, et al. Randomized controlled trial of forward-planned intensity modulated radiotherapy for early breast cancer: interim results at 2 years. Int J Radiat Oncol Biol Phys. 2012;82(2):715-723.

25. Donovan E, Bleakley N, Denholm E, et al; Breast Technology Group. Randomised trial of standard 2D radiotherapy (RT) versus intensity modulated radiotherapy (IMRT) in patients prescribed breast radiotherapy. Radiother Oncol. 2007;82(3):254-264.

26. Pignol JP, Olivotto I, Rakovitch E, et al. A multicenter randomized trial of breast intensity-modulated radiation therapy to reduce acute radiation dermatitis. J Clin Oncol. 2008;26(13):2085-2092.

27. Mukesh MB, Qian W, Wilkinson JS, et al. Patient reported outcome measures (PROMs) following forward planned field-in field IMRT: results from the Cambridge Breast IMRT trial. Radiother Oncol. 2014;111(2):270-275. 
28. Mukesh MB, Barnett GC, Wilkinson JS, et al. Randomized controlled trial of intensity-modulated radiotherapy for early breast cancer: 5-year results confirm superior overall cosmesis. J Clin Oncol. 2013;31(36):4488-4495.

29. Haciislamoglu E, Colak F, Canyilmaz E, et al. Dosimetric comparison of left-sided whole-breast irradiation with 3DCRT, forward-planned IMRT, inverse-planned IMRT, helical tomotherapy, and volumetric arc therapy. Phys Med. 2015;31(4):360-367.

30. Small K, Kelly C, Beldham-Collins R, Gebski V. Whole breast and excision cavity radiotherapy plan comparison: conformal radiotherapy with sequential boost versus intensity-modulated radiation therapy with a simultaneously integrated boost. J Med Radiat Sci. 2013; 60(1):16-24.

31. Al-Rahbi ZS, Ravichandran R, Binukumar JP, Davis CA, Satyapal N, Al-Mandhari Z. A dosimetric comparison of radiotherapy techniques in the treatment of carcinoma of breast. J Cancer Ther. 2013;4(11): 10-17.

32. Smith W, Menon G, Wolfe N, Ploquin N, Trotter T, Pudney D. IMRT for the breast: a comparison of tangential planning techniques. Phys Med Biol. 2010;55(4):1231-1241.
33. George R, Keall PJ, Kini VR, et al. Quantifying the effect of intrafraction motion during breast IMRT planning and dose delivery. Med Phys. 2003;30(4):552-562.

34. Jain P, Marchant T, Green M, et al. Inter-fraction motion and dosimetric consequences during breast intensity-modulated radiotherapy (IMRT) Radiother Oncol. 2009;90(1):93-98.

35. Alford SL, Prassas GN, Vogelesang CR, Leggett HJ, Hamilton CS. Adjuvant breast radiotherapy using a simultaneous integrated boost: clinical and dosimetric perspectives. J Med Imaging Radiat Oncol. 2013;57(2):222-229.

36. Askoxylakis V, Jensen AD, Häfner MF, et al. Simultaneous integrated boost for adjuvant treatment of breast cancer-intensity modulated vs. conventional radiotherapy: the IMRT-MC2 trial. BMC Cancer. 2011;11:249.

37. Chen Z, King W, Pearcey R, Kerba M, Mackillop W. The relationship between waiting time for radiotherapy and clinical outcomes: a systematic review of the literature. Radiother Oncol. 2008;87(1):3-16.

38. Teh BS, Lu HH, Sobremonte S, et al. The potential use of intensity modulated radiotherapy (IMRT) in women with pectus excavatum desiring breast-conserving therapy. Breast J. 2001;7(4):233-239.
Breast Cancer - Targets and Therapy

\section{Publish your work in this journal}

Breast Cancer - Targets and Therapy is an international, peerreviewed open access journal focusing on breast cancer research, identification of therapeutic targets and the optimal use of preventative and integrated treatment interventions to achieve improved outcomes, enhanced survival and quality of life for the cancer patient.

\section{Dovepress}

The manuscript management system is completely online and includes a very quick and fair peer-review system, which is all easy to use. Visit http://www.dovepress.com/testimonials.php to read real quotes from published authors. 